\title{
A Síndrome de Burnout e a assistência de enfermagem em Unidade de Terapia Intensiva diante da pandemia de COVID-19
}

Burnout Syndrome and nursing care in the Intensive Care Unit in view of the COVID-19 pandemic Sindrome de Burnout y cuidados de enfermaria em la Unidad de Cuidados Intensivos ante la pandemia COVID-19

Ana Luiza Gonçalves da Silva ORCID: https://orcid.org/0000-0002-6889-6726 Universidade Paulista, Brasil

E-mail: analuizasntg2@gmail.com

Laiane Magalhães da Silva

ORCID: https://orcid.org/0000-0001-6625-7392 Universidade Paulista, Brasil

E-mail: laianemagalhaes218@gmail.com

Silvania Cristina Pereira Martins ORCID: https://orcid.org/0000-0001-7944-0727 Universidade Paulista, Brasil

E-mail: silp.pereira@gmail.com Joseane Ruas Campos

ORCID: https://orcid.org/0000-0003-2568-7315 Universidade Paulista, Brasil

E-mail: josyaneamj@gmail.com

Lucilene dos Santos Paiva

ORCID: https://orcid.org/0000-0001-6066-1618 Universidade Paulista, Brasil

E-mail: lu.paivasousa@gmail.com

Maria Luíza Rêgo Bezerra

ORCID: https://orcid.org/0000-0002-3336-7760 Universidade Paulista, Brasil

E-mail: maria.bezerra@docente.unip.br

\section{Resumo}

O objetivo é refletir a partir de uma revisão científica nacional e internacional sobre a magnitude da Síndrome de Burnout em enfermeiros que trabalham em Unidade de Terapia Intensiva no contexto da pandemia de Coronavirus Disease 2019 (COVID-19). Trata-se de uma revisão integrativa da literatura, realizada a partir da busca por artigos completos e publicados nas bases de dados: Literatura Latino-Americana e do Caribe em Ciências da Saúde, Biblioteca Eletrônica Científica Online, Bases de Dados de Enfermagem e Medical Literature Analysis and Retrievel System Online, publicados entre 2019 a 2021. Os resultados foram incluídos em dez publicações na amostra final do estudo. Verificou-se a tendência dos enfermeiros à progressão da Síndrome de Burnout durante uma pandemia, como da COVID-19; a princípio o acometimento ou aumento dos sintomas surgem no dia a dia, culminando em um estado grave de exaustão física e emocional que acarreta no surgimento e avanço da síndrome no decorrer do tempo. Podemos concluir que em relação a magnitude da Síndrome de Burnout em enfermeiros que trabalham em Unidade de Terapia Intensiva no contexto da pandemia da COVID-19, tais profissionais apresentaram maior prevalência da Síndrome de Burnout quando comparado a outros setores.

Palavras-chave: Esgotamento profissional; Enfermagem; COVID-19; Unidades de Terapia Intensiva; Saúde mental.

\begin{abstract}
The objective is to reflect based on a national and international scientific review on the magnitude of Burnout Syndrome in nurses working in the Intensive Care Unit in the context of the Coronavirus 2019 Disease pandemic (COVID-19). This is an integrative literature review, based on a search for complete articles published in the following databases: Latin American and Caribbean Literature in Health Sciences, Online Scientific Electronic Library, Nursing Databases and Medical Literature Analysis and Retrievel System Online, published between 2019 and 2021. The results were included in ten publications in the final study sample. There was a tendency for nurses to progress with Burnout Syndrome during a pandemic, such as COVID-19; at first, the onset or increase of symptoms appears on a daily basis, culminating in a severe state of physical and emotional exhaustion that leads to the emergence and advancement of the
\end{abstract}


syndrome over time. We can say that in relation to the magnitude of Burnout Syndrome in nurses working in the Intensive Care Unit in the context of the COVID-19 pandemic, such superior professionals are higher in Burnout Syndrome when compared to other sectors.

Keywords: Burnout professional; Nursing; COVID-19; Intensive Care Units; Mental health.

\section{Resumen}

El objetivo es reflexionar a partir de una revisión científica nacional e internacional sobre la magnitud del Síndrome de Burnout en enfermeras que laboran en la Unidad de Cuidados Intensivos en el contexto de la Pandemia de Enfermedad por Coronavirus 2019 (COVID-19). Se trata de una revisión integradora de la literatura, basada en una búsqueda de artículos completos publicados en las siguientes bases de datos: Literatura Latinoamericana y del Caribe en Ciencias de la Salud, Biblioteca Electrónica Científica en Línea, Bases de Datos de Enfermería y Análisis de la Literatura Médica y Retrievel System Online, publicados entre 2019 y 2021 Los resultados se incluyeron en diez publicaciones de la muestra final del estudio. Hubo una tendencia de las enfermeras a progresar con el síndrome de Burnout durante una pandemia, como COVID-19; En un primer momento, la aparición o el aumento de los síntomas se manifiesta a diario, culminando en un severo estado de agotamiento físico y emocional que conduce al surgimiento y avance del síndrome a lo largo del tiempo. Podemos decir que en relación a la magnitud del Síndrome de Burnout en los enfermeros que laboran en la Unidad de Cuidados Intensivos en el contexto de la pandemia de COVID-19, estos profesionales superiores son más altos en Síndrome de Burnout en comparación con otros sectores.

Palabras clave: Agotamiento profesional; Enfermería; COVID-19; Unidades de Cuidados Intensivos; Salud mental.

\section{Introdução}

A pandemia de COVID-19, ocasionada pelo novo coronavírus, é uma infecção respiratória provocada pelo Coronavírus da Síndrome Respiratória Aguda Grave 2 (SARS-CoV-2). Foi reportada na Província de Whuan, na China, no início de dezembro de 2019. A rápida transmissibilidade do vírus em diversos países, em janeiro de 2020, fez com que a Organização Mundial de Saúde (OMS), em 11 de março de 2020, considerasse a COVID-19 uma pandemia de emergência em saúde pública de Importância Internacional (ESPII) (Santos et al., 2021). Com o aflorar do novo cenário mundial, impactos na área da saúde vêm trazendo preocupação, especialmente, no que concerne à saúde mental e física dos profissionais na linha de frente do atendimento às vítimas do coronavírus (Teixeira et al., 2020).

A crescente incidência de casos, devido ao avanço da COVID-19, superlotou os serviços de saúde pública e, especialmente, os serviços de Unidade de Terapia Intensiva (UTI), sendo apontado como um grande desafio no setor público pela escassez de leitos e suprimentos (Santos et al., 2021). Nos ambientes hospitalares, a UTI é considerada um setor com máximo nível de estresse ocupacional, o que tem favorecido o adoecimento mental e o esgotamento físico dos profissionais que ali trabalham. Certamente, pelo contexto da pandemia, problemas que já eram recorrentes na UTI, como a alta demanda de atividades, quantidade de profissionais reduzida, pacientes críticos, escassez de insumos e ambiente inapropriado, têm contribuído para a sobrecarga de trabalho e com o quadro de sofrimento psicofísico dos profissionais (Maziero et al., 2019).

Diante desse contexto, os sistemas de saúde dos países afetados entraram em colapso, e os enfermeiros envolvidos direta e indiretamente no enfrentamento da pandemia estão cada vez mais exaustos devido aos longos turnos de trabalho e ao distanciamento social - medida adotada para reduzir a disseminação da doença. Além da exposição constante e do risco de adoecimento pelo novo coronavírus, é evidente que, em vista desse cenário, o enfermeiro tende a desenvolver desgaste no que concerne a sua saúde mental e física; concomitantemente há o surgimento de problemas como: estresse crônico, cansaço físico, transtorno de ansiedade, sono prejudicado, Síndrome de Burnout (SB) e depressão (Brooks et al., 2020).

A SB é um fenômeno psicossocial desencadeado pelo estresse laboral, gerando consequências negativas em âmbitos social, profissional e familiar. De origem inglesa, a palavra Burnout é composta por duas partes: burn refere-se à "queima" e out quer dizer "exterior", ratificando sintomas em pessoas acometidas pela síndrome: diminuição ou perda de particularidades mentais, emocionais e físicas, que é originada por modificações entre o indivíduo e o ambiente, referindo-se a algo que deixou de fazer por exaustão (Ramos et al., 2019). 
Com o surgimento da pandemia, agravou ainda mais o cenário desfavorável dos enfermeiros, e o esgotamento profissional se tornou mais recorrente, afinal, antes mesmo de instalar-se a crise nos serviços de saúde por conta da COVID-19, tais profissionais já sofriam os efeitos da precariedade na saúde, pois enfrentavam graves problemas estruturais, organizacionais e laborais, além de desempenharem suas tarefas em setores complexos que demandam diferentes graus de exigências da profissão, requerendo eficiência, aptidão e embasamento científico atualizado (Bao et al., 2020).

Os enfermeiros atuam e estão amplamente comprometidos com as relações humanas, fato exigido pela profissão. Dada a complexidade de seus relacionamentos interpessoais, intraprofissionais e multiprofissionais, associados às atividades laborais, observa-se um aumento de doenças devido ao esgotamento físico-mental (Silva et al., 2018).

Dessa forma, elaborou-se a seguinte pergunta-problema: Como a Síndrome de Burnout na assistência de enfermagem em Unidade de Terapia Intensiva diante da COVID-19 vem sendo retratado nas publicações científicas nacionais e internacionais de enfermagem?

O presente estudo tem como objetivo refletir a partir de uma revisão científica nacional e internacional sobre a magnitude da Síndrome de Burnout em enfermeiros que trabalham em Unidade de Terapia Intensiva no contexto da pandemia de COVID-19.

\section{Metodologia}

A seleção do tipo metodológico é fundamental para iniciar a construção e organização de uma pesquisa, bem como para traçar os modelos de coleta de dados e a observação do conteúdo.

\section{a) Tipo de estudo}

Trata-se de uma revisão integrativa da literatura no que se refere à SB em profissionais de enfermagem atuantes na Unidade de Terapia Intensiva no contexto da pandemia de COVID-19. A finalidade deste método de pesquisa é analisar estudos anteriores e propiciar uma vasta compreensão sobre um assunto específico. Essa estratégia de pesquisa proporciona a síntese de inúmeros artigos e contribui para condensar resultados de estudos referentes ao mesmo tema (Mendes et al., 2019).

\section{b) Procedimento metodológico}

Com relação à composição desta pesquisa, foram utilizadas as seguintes etapas para sua elaboração: 1) construção da pergunta norteadora da pesquisa; 2) levantamento e seleção das publicações nas bases de dados selecionadas; 3) extração de dados dos artigos selecionados; 4) análise crítica dos estudos escolhidos; 5) apresentação dos resultados encontrados e 6) apresentação do método (Mendes et al., 2019).

\section{c) Coleta e organização dos dados}

Para o levantamento dos artigos na literatura, foi realizada uma busca na base de dados da Literatura Latino-Americana e do Caribe em Ciências da Saúde (LILACS), Biblioteca Eletrônica Científica Online (SCIELO), Bases de Dados de Enfermagem (BDENF) e Medical Literature Analysis and Retrievel System Online (MEDLINE). Foi utilizada para busca dos artigos, os seguintes descritores em ciências da saúde $(\mathrm{DeCs})$, que deveriam estar no título e/ou no resumo: Esgotamento profissional; Enfermagem; Covid-19; Unidades de Terapia Intensiva e saúde mental.

Os descritores foram combinados entre si por meio do operador booleano " $A N D$ " que originou as estratégias de busca descritas abaixo (Quadro 1): 
Quadro 1 - Estratégia de Busca - Brasília-DF - 2021.

\begin{tabular}{|c|c|}
\hline LILACS & $\begin{array}{l}\text { (Esgotamento profissional and COVID-19); (saúde mental and COVID-19); (unidade de } \\
\text { terapia intensiva and COVID-19); (unidade de terapia intensiva and saúde mental); } \\
\text { (unidade de terapia intensiva and esgotamento profissional); (enfermagem and } \\
\text { esgotamento profissional); (enfermagem and COVID-19); (enfermagem and unidade de } \\
\text { terapia intensiva). }\end{array}$ \\
\hline BDENF & $\begin{array}{l}\text { (Esgotamento profissional and COVID-19); (saúde mental and COVID-19); (unidade de } \\
\text { terapia intensiva and COVID-19); (unidade de terapia intensiva and saúde mental); } \\
\text { (unidade de terapia intensiva and esgotamento profissional); (enfermagem and } \\
\text { esgotamento profissional); (enfermagem and COVID-19); (enfermagem and unidade de } \\
\text { terapia intensiva). }\end{array}$ \\
\hline MEDLINE & $\begin{array}{l}\text { (Esgotamento profissional and COVID-19); (saúde mental and COVID-19); (unidade de } \\
\text { terapia intensiva and COVID-19); (unidade de terapia intensiva and saúde mental); } \\
\text { (unidade de terapia intensiva and esgotamento profissional); (enfermagem and } \\
\text { esgotamento profissional); (enfermagem and COVID-19); (enfermagem and unidade de } \\
\text { terapia intensiva). }\end{array}$ \\
\hline SCIELO & $\begin{array}{l}\text { (Esgotamento profissional and COVID-19); (saúde mental and COVID-19); (unidade de } \\
\text { terapia intensiva and COVID-19); (unidade de terapia intensiva and saúde mental); } \\
\text { (unidade de terapia intensiva and esgotamento profissional); (enfermagem and } \\
\text { esgotamento profissional); (enfermagem and saúde mental); (enfermagem and COVID- } \\
\text { 19); (enfermagem and unidade de terapia intensiva). }\end{array}$ \\
\hline
\end{tabular}

Fonte: Autores (2021).

Os artigos foram organizados a partir das características dos estudos selecionado: 1) autor e ano de publicação; 2) título do artigo; 3 ) periódico; 4) método do estudo e 5) nível de evidência. Os níveis de evidência foram classificados pelo tipo de estudo do artigo, por meio da pirâmide do nível de evidência nas ciências médicas: I) metanálises; II) revisões sistemáticas; III) ensaios clínicos randomizados; IV) coorte caso- controle; V) estudo transversal relato de caso. O quadro detalhado das etapas está apresentado a seguir no quadro 2 dos resultados (Montagna et al., 2020).

\section{d) Análise dos Dados}

A partir da coleta de dados, localizaram-se 1.919 artigos que foram submetidos à primeira fase de análise por meio de critérios de inclusão e exclusão anteriormente definidos na norma de pesquisa. Para a seleção dos artigos, os critérios de inclusão adotados foram: artigos originais publicados nos períodos de 2019 a 2021, textos completos disponíveis nas bases de dados selecionadas, nos idiomas português, inglês e espanhol, condizentes com a temática proposta e com os descritores listados. Foram excluídos automaticamente artigos científicos duplicados entre as bases de dados, artigos de revisão integrativa de literatura e relatos de experiência. Essa etapa foi realizada a partir da leitura do resumo de cada artigo e, quando necessário, da consulta ao texto na íntegra.

Deste modo, obteve-se um total de 68 artigos ao final da primeira fase, dos quais 20(29,41\%) foram encontrados na LILACS, 26(38,23\%) na SciELO, 12(17,64\%) na BDENF e 10 na MEDLINE (14,70\%).

Na etapa seguinte, procedeu-se à análise integral dos 68 artigos, a fim de identificar os artigos que cumpriam com os critérios de inclusão do estudo previamente definidos. Após a seleção, adquiriu-se uma amostra de 13 artigos, destes, 7(53,84\%) da SciELO, 3(23,07\%) LILACS, 1(7,69\%) BDENF e 2(15,38\%) MEDLINE. O fluxograma com o detalhamento das etapas de pesquisa está apresentado a seguir na Figura 1. 
Figura - Fluxograma coleta e análise dos dados - Brasília-DF - 2021.

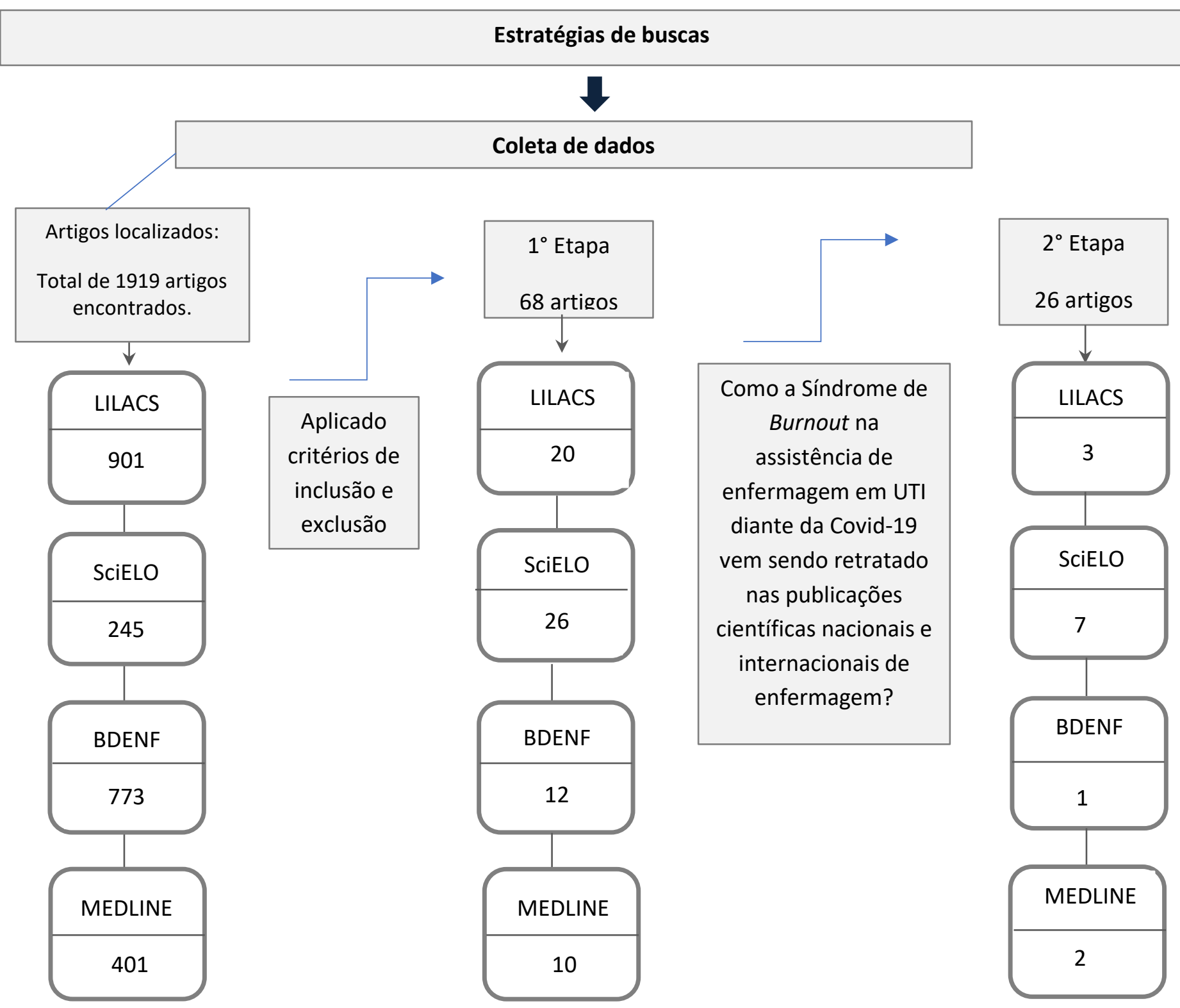

Fonte: Autores (2021).

\section{Resultados}

Apresentaremos o Quadro 2 com os dados dos 13 artigos selecionados, sendo esses referentes a SB, UTI e COVID-19, descrevendo assim as características dos estudos elegíveis. 
Quadro 2 - Características dos estudos selecionados - Brasília-DF - 2021.

\begin{tabular}{|c|c|c|c|c|c|}
\hline $\mathrm{N}^{\mathrm{o}}$ & Autores e ano & Título & Periódico & Método & $\begin{array}{l}\text { Nível de } \\
\text { evidencia }\end{array}$ \\
\hline 1 & $\begin{array}{l}\text { HORTA LR, et al., } \\
\qquad(2021)\end{array}$ & $\begin{array}{c}\text { O estresse e a saúde mental de profissionais } \\
\text { da linha de frente da COVID-19 em hospital } \\
\text { geral. }\end{array}$ & $\begin{array}{l}\text { Jornal Brasileiro de } \\
\text { Psiquiatria }\end{array}$ & Transversal & IV \\
\hline 2 & $\begin{array}{l}\text { ARAGÃO CSN, et al., } \\
\qquad(2021)\end{array}$ & $\begin{array}{c}\text { Síndrome de Burnout e Fatores Associados } \\
\text { em Enfermeiros de Unidade de Terapia } \\
\text { Intensiva. }\end{array}$ & $\begin{array}{l}\text { Revista Brasileira } \\
\text { de Enfermagem }\end{array}$ & Transversal & IV \\
\hline 3 & $\begin{array}{l}\text { ALVES CCM, et al., } \\
\qquad(2021)\end{array}$ & $\begin{array}{l}\text { Prevalência de esgotamento profissional em } \\
\text { técnicos em enfermagem de uma unidade de } \\
\text { Terapia Intensiva Adulto. }\end{array}$ & $\begin{array}{l}\text { Revista Brasileira } \\
\text { de Enfermagem }\end{array}$ & Transversal & IV \\
\hline 4 & $\begin{array}{l}\text { SÁNCHEZ-SÁNCHEZ } \\
\text { E, et al., (2021) }\end{array}$ & $\begin{array}{c}\text { Impact of the COVID-19 Pandemic on the } \\
\text { Mental Health of Nurses and Auxiliary } \\
\text { Nursing Care Technicians-A Voluntary } \\
\text { Online Survey. }\end{array}$ & $\begin{array}{l}\text { Int J Environ Res } \\
\text { Public Health }\end{array}$ & Transversal & V \\
\hline 5 & $\begin{array}{l}\text { DUARTE CM, et al., } \\
\text { (2021) }\end{array}$ & $\begin{array}{l}\text { Enfermagem e saúde mental: uma reflexão } \\
\text { em meio à pandemia de coronavírus. }\end{array}$ & $\begin{array}{l}\text { Revista Gaúcha de } \\
\text { enfermagem }\end{array}$ & $\begin{array}{l}\text { Teórico- } \\
\text { reflexivo }\end{array}$ & II \\
\hline 6 & $\begin{array}{l}\text { MOTA RS, et al., } \\
(2021)\end{array}$ & $\begin{array}{c}\text { Estresse ocupacional relacionado à } \\
\text { assistência de enfermagem em terapia } \\
\text { intensiva. }\end{array}$ & $\begin{array}{l}\text { Revista Baiana de } \\
\text { Enfermagem }\end{array}$ & Transversal & III \\
\hline 7 & $\begin{array}{l}\text { MORENO MC, et al., } \\
(2021)\end{array}$ & $\begin{array}{c}\text { The Impact of the COVID-19 Pandemic on } \\
\text { ICU Healthcare Professionals: A Mixed } \\
\text { Methods Study. }\end{array}$ & $\begin{array}{l}\text { Int J Environ Res } \\
\text { Public Health }\end{array}$ & Misto & II \\
\hline 8 & $\begin{array}{l}\text { DAL'BOSCO EB, et } \\
\text { al., (2020) }\end{array}$ & $\begin{array}{l}\text { A saúde mental da enfermagem no } \\
\text { enfrentamento da COVID-19 em um hospital } \\
\text { universitário regional. }\end{array}$ & $\begin{array}{l}\text { Revista Brasileira } \\
\text { de Enfermagem }\end{array}$ & Transversal & $\mathrm{V}$ \\
\hline 9 & $\begin{array}{l}\text { SILVA APF, et al., } \\
(2020)\end{array}$ & $\begin{array}{c}\text { Incidência da Síndrome de Burnout em } \\
\text { profissionais de enfermagem atuantes em } \\
\text { unidade de terapia intensiva. }\end{array}$ & $\begin{array}{l}\text { Revista de Pesquisa } \\
\text { (Univ. Fed. Estado } \\
\text { Rio J) }\end{array}$ & $\begin{array}{l}\text { Pesquisa de } \\
\text { Campo }\end{array}$ & III \\
\hline 10 & $\begin{array}{l}\text { ALVARES MEM, et } \\
\text { al., (2020) }\end{array}$ & $\begin{array}{l}\text { Síndrome de burnout entre profissionais de } \\
\text { saúde nas unidades de terapia intensiva: um } \\
\text { estudo transversal com base populacional. }\end{array}$ & $\begin{array}{l}\text { Revista Brasileira } \\
\text { de Terapia Intensiva }\end{array}$ & Transversal & IV \\
\hline 11 & $\begin{array}{l}\text { DUTRA HS, et al., } \\
(2019)\end{array}$ & $\begin{array}{l}\text { Burnout entre profissionais de enfermagem } \\
\text { em hospitais noBrasil. }\end{array}$ & Revista Cuidarte & Transversal & III \\
\hline 12 & $\begin{array}{l}\text { BEZERRA CMB, et } \\
\text { al., (2019) }\end{array}$ & $\begin{array}{l}\text { Prevalência do estresse e síndrome de } \\
\text { burnout em enfermeiros no trabalho } \\
\text { hospitalar em turnos. }\end{array}$ & $\begin{array}{l}\text { REME } \\
\text { ver.min.enferm }\end{array}$ & $\begin{array}{l}\text { Pesquisa de } \\
\text { campo }\end{array}$ & III \\
\hline 13 & $\begin{array}{l}\text { SILVA AA, et al., } \\
(2019)\end{array}$ & $\begin{array}{c}\text { Variáveis preditoras para burnout entre } \\
\text { profissionais de enfermagem }\end{array}$ & $\begin{array}{l}\text { Revista de } \\
\text { Psicologia }\end{array}$ & Transversal & IV \\
\hline
\end{tabular}

Fonte: Autores (2021).

\section{a) Descrição dos Estudos}

A evolução dos estudos analisados perfez uma trajetória de 2019 a 2021 , notou-se que os 13 artigos analisados foram publicados em 10 periódicos diferentes. O periódico com maior número de artigos selecionados foi a Revista Brasileira de Enfermagem, com 3(30\%) publicações, seguido da Int J Environ Res Public Health com 2(20\%). Do total de 13 artigos selecionados, o ano de 2021 apresentou 53,84\% das publicações no período analisado, seguido do ano de 2020 com 23,07\% 7 e, por fim, o ano 2019 com 23,07\% dos artigos selecionados. No que se refere à abordagem metodológica, 9(69,23\%) publicações eram estudos transversais, 2(15,38\%) pesquisa de campo, 1(7,69\%) misto e 1(7,69\%) teórico reflexivo. Em relação ao nível de evidência, foram identificados 5(38,46\%) artigos de nível IV, 4(30,76\%) artigos de nível III e 2(15,38\%) artigo de nível V e 2(15,38\%) artigo de nível II. 


\section{b) Descrição dos Resultados}

Em estudo realizado por Dutra et al. (2019) acerca das alterações emocionais, dentre os 452 profissionais de enfermagem entrevistados no estado de Minas Gerais, a maioria deles apresentou níveis baixos de Burnout quando relacionado às dimensões, com nível baixo na exaustão emocional $(38,94 \%)$ e despersonalização $(45,80 \%)$ e níveis moderados $(39,16 \%)$ na realização pessoal.

Para Sánchez et al. (2021), na pandemia, no que concerne a organização do trabalho, a ineficaz percepção de suporte organizacional ocasionada, pela falta de recursos materiais e equipamentos de proteção individual (EPIs), influenciou na saúde mental dos enfermeiros. Um estudo transversal realizado, usando um questionário anônimo e autoaplicável entre profissionais de enfermagem, mostrou que a maior carga psicológica durante o primeiro período da pandemia adveio da falta de EPIs (40,0\%), medo de infectar seus familiares $(25,3 \%)$ e falta de protocolos $(14,8 \%)$.

Em relação aos aspectos da assistência de enfermagem, um estudo conduzido entre junho e novembro nas ilhas Baleares, Espanha, demonstrou que em relação ao atendimento aos familiares dos pacientes críticos, dos enfermeiros entrevistados (135\%) apresentaram maior grau de desgaste e (113\%) apresentaram desgaste em relação à assistência das necessidades emocionais dos familiares (Mota et al., 2021).

\section{Discussão}

Os artigos selecionados trazem um conjunto de fatores que impactam diretamente na saúde mental dos enfermeiros de UTI que estão à frente no combate à COVID-19. Desde o início, os enfermeiros que estão na linha de frente dessa pandemia foram submetidos a uma alta carga emocional que influenciou negativamente sua saúde mental, decorrente de uma combinação de estressores no local de trabalho e medos pessoais. Estudos demonstraram um aumento do sofrimento moral, prevalência de níveis de estresse moderado ou alto, Burnout, ansiedade, depressão e maior frequência de sinais de exaustão, resultados do potencial exposição dos enfermeiros frente à pandemia (Horta et al., 2021).

De acordo com Aragão et al. (2021), a SB é um transtorno mental caracterizado por tensão emocional e esgotamento causado por condições estressantes de trabalho, além disso, é definida por exaustão física, psicológica e emocional, podendo abranger indivíduos de diferentes categorias profissionais, gêneros e faixas etárias, mas, principalmente, aqueles que desenvolvem atividades que requerem relacionar-se de forma direta e prolongada com o outro, como os enfermeiros de UTI.

Alvares et al. (2020) explicam que essa síndrome inclui três fatores dimensionais e interdependentes: a exaustão emocional, a despersonalização e a baixa realização profissional. A exaustão emocional é conceituada como um esgotamento das emoções e sentimentos para resistir às vivências do cotidiano e acarretam mudanças físicas, mentais e também declínio da produção no meio laboral, já a despersonalização é caracterizada pela instabilidade emocional do profissional, que causa afastamento e conduta rude e indesejável com os pacientes e colegas de trabalho, a baixa satisfação no trabalho é definida pela incapacidade de perceber suas habilidades e empenho como um ato assertivo e só se auto avaliar negativamente gerando insatisfação e infelicidade profissional.

Os indícios clínicos característicos do Burnout podem ser observados em manifestações, de maneira simultânea ou isoladamente, as quais se referem aos sintomas físicos ou psicossomáticos, que estão relacionados à fadiga diária, a dores musculares, a alterações nos padrões que envolvem o sono e os distúrbios do apetite; sintomas psíquicos, que resultam em frustrações e alteração no humor; sintomas emocionais, nos quais é notório a redução do rendimento, concentração laboral e o surgimento de sintomas de ansiedade; e sintomas comportamentais e defensivos, que estão ligados à forte tendência ao isolamento social e sentimentos de irritabilidade (Alves et al., 2021).

Com a exposição diária a pacientes com COVID-19, sucedeu um aumento no número de profissionais afastados de suas funções após serem infectados pelo novo coronavírus, por consequência ocorreu uma progressão de casos de distúrbios do sono 
entre os enfermeiros no início da pandemia, devido a diversos fatores, como a não contratação de profissionais e redução de equipe na UTI, exigindo de alguns profissionais turnos extras, o que acarretou prejuízo considerável no sono dos enfermeiros, aumento da insônia ou frequência das interrupções do sono (Sánchez et al., 2021).

O atual cenário exigiu muito dos enfermeiros, bem como dos serviços de saúde, levando-os a se reinventarem e a reorganizarem suas atividades laborais: priorizar pacientes de urgência e emergência, adiar consultas e procedimentos eletivos, destinar atenção prioritária aos atendimentos do novo coronavírus para o enfrentamento da pandemia (Duarte et al., 2021).

Dal'Bosco et al. (2020) argumenta que a predominância da SB em UTI vem ganhando força nos últimos anos, e a literatura mostra que a ansiedade é mais evidente entre os profissionais de saúde e pior entre os enfermeiros que atuam em setores críticos, pois, além dos conflitos e pressões cotidianas que exigem tomadas de decisão rápida, eles também enfrentam questões de natureza física, química e biológica. No cenário da pandemia, a grande parcela dos pacientes acometidos pela COVID-19 manifesta sintomas graves da doença, sobretudo, síndromes respiratórias, necessitando de cuidados intensivos e de recursos tecnológicos avançados para a monitorização constante de seus parâmetros vitais, o que justifica maior prevalência de ansiedade em enfermeiros atuantes em setores críticos.

A atuação dos enfermeiros intensivistas frente à COVID-19 e à grande demanda, nessa área de casos de alta complexidade - UTI enfatizou condições estressantes já existentes e que os expõem a riscos ocupacionais, sendo estes: risco físico (estrutura, temperatura do ambiente, iluminação), químico (medicamento), psicossocial (estresse, fadiga, insatisfação), biológico (micro-organismos) e ergonômico (postura incorreta, excesso de peso), além de ser um local que oferece risco de transmissão de infecções em decorrência da assistência oferecida a pacientes graves e risco de contaminação devido a procedimentos cirúrgicos (Silva et al., 2020). Dessa forma, acredita-se que as especificidades dispostas no meio laboral e a falta de gerenciamento dos fatores desordenados e estressantes, bem como a ausência de possibilidade de mudanças, exigentes cobranças, obrigações e conflitos permanentes, por conseguinte, podem causar danos psicológicos e acarretar Burnout (Bezerra et al., 2019).

A falta de infraestrutura e organização do setor atrapalha na execução das atividades práticas dos enfermeiros e geram intercorrências impróprias, às vezes, pela falta de autonomia profissional, escassez de insumos, má distribuição de funcionários, ausência de feedback ou falta de incentivo aos profissionais, levando-os ao descontentamento profissional e ao declínio do bemestar pessoal. Sendo assim, é essencial que o profissional mantenha sua autonomia, domínio sobre o meio laboral e uma base segura e organizacional (Silva et al., 2019).

Paralelo a isso, entre as condições relacionadas às práticas de trabalho dos enfermeiros (tornando-os propensos ao Burnout), destaca-se a dificuldade nas relações entre os enfermeiros e familiares dos pacientes decorrente da falta de compreensão e reconhecimento do trabalho de enfermagem pela família (Mota et al., 2021). Por fim, segundo Moreno et al. (2021), vale ressaltar que o isolamento durante a pandemia agravou ainda mais a exaustão e o estresse emocional dos enfermeiros, pois em circunstâncias normais esses profissionais podiam buscar conforto na vida familiar e social para lidar com o estresse ocupacional.

\section{Conclusão}

Podemos concluir que em relação a magnitude da SB em enfermeiros que trabalham em UTI no contexto da pandemia da COVID-19, tais profissionais apresentaram maior prevalência da SB quando comparado a outros setores, além disso, este estudo buscou identificar e refletir sobre os fatores que têm contribuído para o agravamento dessa síndrome durante a pandemia da COVID-19. O interesse neste assunto surgiu em razão dos dados científicos, que reconhecem as implicações na saúde mental dos enfermeiros, antes e durante a pandemia, e comprovam que esses profissionais ocupam posição estressante no meio laboral, 
sobretudo, na UTI, decorrente da sobrecarga de trabalho, escassez de insumos e recursos humanos, responsabilidade técnica inerente da profissão, além de condições de risco que colaboram com o surgimento de quadros de ansiedade, exaustão emocional, depressão, estresse e desenvolvimento da SB.

O bem-estar e a saúde mental dos enfermeiros são fundamentais para uma assistência livre de erros que possam retardar a recuperação do paciente ou levá-lo à morte. Nessa perspectiva, para uma boa saúde mental dos enfermeiros, devem ser realizadas estratégias que minimizem os episódios de estresse e se concentrem na resolubilidade real dos problemas, conforme a necessidade demonstrada no local de trabalho, além do dimensionamento profissional e divisão das atividades de forma igualitária. Diante disso, é importante garantir que o enfermeiro receba assistência psicológica de forma a zelar pela saúde ocupacional de cada indivíduo dentro e fora do seu ambiente de trabalho.

\section{Referências}

Aragão, C. S. N., Barbosa, B. G., Cleide Lucilla Carneiro Santos, C. L. C., Nascimento, S. S. D., Bôas, V. B. L., Júnior, M. F. D., \& Sobrinho, N. L. C. (2021). Síndrome de Burnout e Fatores Associados em Enfermeiros de Unidade de Terapia Intensiva. Rev. Bras. Enferm. 74(3).

Alvares, M. E. M., Thomaz, F. E., Lamy, C. Z., Nina, H. R., Pereira, L. M., \& Garcia, S. J. (2020). Síndrome de burnout entre profissionais de saúde nas unidades de terapia intensiva: um estudo transversal com base populacional. Rev. bras. ter. intensiva 32(2).

Alves, C. C. M., Barilli, S. L. S., Specht, M. A., \& Herbert, R. D. N. (2021). Prevalência de esgotamento profissional em técnicos em enfermagem de uma unidade de Terapia Intensiva Adulto. Rev. Bras. Enferm., 74 (3).

Bezerra, C. M. B., Silva, K. K. M., Costa, J. W. S., Farias, J. C., Martino, M. M. F., \& Medeiros, S. M. (2019). Prevalência do estresse e síndrome de burnout em enfermeiros no trabalho hospitalar em turnos. REME rev. min. enferm ; 23: e-1232, jan.

Brooks, K. S., Webster, K. R., Smith, E. L., Woodland, L., Wessely, S., Greenberg, N., \& Rubin, J. G. (2020). The psychological impact of quarantine and how to reduce it: rapid review of the evidence. Mar 14;395(10227), 912-920.

Bao, Y., Sun, Y., Meng, S., Shi, J., \& Lu, L. (2020). 2019-n. CoV epidemic: address mental health care to empower society. Feb 22;395(10224, 37-38.

Duarte, C. M., Silva, G. D., Bagatini, C. M. (2021). Enfermagem e saúde mental: uma reflexão em meio à pandemia de coronavírus. Rev. Gaúcha Enferm. 42.

Dal'Bosco, E. B., Floriano, L. S. M., Skupien, S. V., Arcaro, G., Martins, A. R., \& Anselmo, A. C. C. (2020). A saúde mental da enfermagem no enfrentamento da COVID-19 em um hospital universitário regional. Rev. bras. enferm; 73(2).

Dutra, H. S., Gomes, P. A. L., Garcia, R. N., Oliveira, H. C., Freitas, S. C., \& Guirardello, E. B. (2019). Burnout entre profissionais de enfermagem em hospitais no Brasil. rev. cuid. (Bucaramanga. 2010); 10(1).

Horta, L. R., Camargo, G. E., Barbosa, L. L. M., Lantin, S. J, P., Sette, G. T., Lucini, G. C. T., Silveira, F. A., Zanini, L., \& Lutzky, A. B. (2021). O estresse e a saúde mental de profissionais da linha de frente da COVID-19 em hospital geral. J. bras. psiquiatr. 70(1).

Maziero, S. C. E., Cruz, A. D. E., Alpendre, T. F., Brandão, B. M., Teixeira, R. F. F., \& Krainski, T. E. (2019). Associação entre condições de trabalho da enfermagem e ocorrência de eventos adversos em Unidades Intensivas neopediátricas. Rev. esc. enferm. USP 54.

Mendes, S. D. K., Silveira, P. C. R., Galvão, M. C. (2019). Uso de gerenciador de referências bibliográficas na seleção dos estudos primários em revisão integrativa. Texto contexto - enferm. 28.

Mota, R. S., Silva, V., A., Brito, I. G., Barros, Â. S., Santos, O. M. B., Mendes, A. S., \& Souza, L. C. Mota, Rosana Santos; Silva, Valdenir Almeida da; Brito, Isadora Goncalves; Barros, Ângela de Souza; Santos, Olga Maria Brito dos; Mendes, Andreia Santos; Souza, Lorena de Carvalho. (2021). Estresse ocupacional relacionado à assistência de enfermagem em terapia intensiva. Rev. baiana enferm ; 35.

Moreno, M. C., Sansó, N., Carrero, P. A. López, D. C., Galiana, L., García, P. P., Borràs, M. M. M., Miró, B. M. (2021). The Impact of the COVID-19 Pandemic on ICU Healthcare Professionals: A Mixed Methods Study. Int J Environ Res Public Health ; 18(17).

Montagna, E., Zaia, V., Laporta, Z. G. (2020). Adoção de protocolos para aprimoramento da qualidade da pesquisa médica. Einstein. 18.

Ramos, C. E. B., Farias, J. A., Costa, M. B. S., Fonseca, L. C. T. (2019). Impactos da Síndrome de Burnout na qualidade de vida dos profissionais de enfermagem da atenção básica à saúde. Rev. bras. ciênc. saúde 23(3): 285-296.

Santos, S. B. T., Andrade, R. L., Vieira, L. S., Duarte, A. J., Martins, S. J., Rosado, B. L., Oliveira, S. J., \& Pinto, M. C. I. (2021). Contingência hospitalar no enfrentamento da COVID-19 no Brasil: problemas e alternativas governamentais. Rev. Bras. Enferm, 74(3).

Sánchez-Sánchez, E., García-Álvarez, J. Á., García-Marín, E., Gutierrez-Serrano, M., Alférez, M. J. M., \& Ramirez-Vargas, G. (2021). Impact of the COVID19 Pandemic on the Mental Health of Nurses and Auxiliary Nursing Care Technicians-A Voluntary Online Survey. Int J Environ Res Public Health; 18(16).

Silva, A. A., Sanchez, M. G., Mambrini, B. S. N., Oliveira, Z. M. (2019). Predictor variables for burnout among nursing profe ssional. Revista de Psicología Vol. 37 (1). 
Research, Society and Development, v. 10, n. 14, e590101422473, 2021

(CC BY 4.0) | ISSN 2525-3409 | DOI: http://dx.doi.org/10.33448/rsd-v10i14.22473

Silva, A. P. F., Carneiro, L. V., Ramalho, J. P. G. (2020). Incidência da Síndrome de Burnout em profissionais de enfermagem atuantes em unidade de terapia intensiva. Rev. Pesqui. (Univ. Fed. Estado Rio J., Online) ; 12: 915-920, jan.-dez.

Silva, G. S. Alves., Silva, G. A. V., Silva, R. M., Andolhe, R., Padilha, K. G., \& Costa, A. L. S. (2018). Estresse e burnout em profissionais de enfermagem de unidade de terapia intensiva e semi-intensiva. REVISA (Online) ; 7(1): 5-11.

Teixeira, S. F. C., Soares, M. C., Souza, A. E., Lisboa, S. E., Pinto, M. C. I., Andrade, R. L., \& Espiridião, A. M. (2020). A saúde dos profissionais de saúde no enfrentamento da pandemia de Covid-19. Ciênc. saúde coletiva 25 (9). 